\title{
The iterative solutions of split common fixed point problem for asymptotically nonexpansive mappings in Banach spaces
}

\author{
Yuanheng Wang ${ }^{1 *}$, Xiuping $\mathrm{Wu}^{1}$ and Chanjuan Pan ${ }^{1}$
}

"Correspondence: yhwang@zinu.cn 'Department of Mathematics, Zhejiang Normal University, Jinhua, 321004 Zhejiang, China

\begin{abstract}
In this paper, we propose an iteration algorithm for finding a split common fixed point of an asymptotically nonexpansive mapping in the frameworks of two real Banach spaces. Under some suitable conditions imposed on the sequences of parameters, some strong convergence theorems are proved, which also solve some variational inequalities that are closely related to optimization problems. The results here generalize and improve the main results of other authors.

Keywords: Split common fixed point; Strong convergence; Variational inequality; Asymptotically nonexpansive mapping
\end{abstract}

\section{Introduction}

Since 1994, the split feasibility problem (SFP) [1-3] has received much attention, owing to its applications in many optimization problems, signal processing and medical image reconstruction with special progress in intensity-modulated radiation therapy [4-6]. Let us recall the SFP: to find a point $q \in B_{1}$ such that

$$
q \in C \quad \text { such that } A q \in Q \text {, }
$$

where $A: B_{1} \longrightarrow B_{2}$ is a bounded linear operator, $C$ and $Q$ are nonempty closed convex subsets of two real Hilbert spaces $B_{1}$ and $B_{2}$, respectively.

It is easy to see that problem (1.1) is equivalent to the following fixed point equation:

$$
u=P_{C}\left(I-\delta A^{*}\left(I-P_{Q}\right) A\right) u, \quad u \in C,
$$

where $A^{*}$ is the corresponding adjoint operator of $A$, the stepsize $\delta$ is a properly chosen real number, and $P_{C}$ and $P_{Q}$ are the metric projections from $B_{1}$ and $B_{2}$ onto $C$ and $Q$, respectively. If $\delta \in\left(0, \frac{2}{\|A\|^{2}}\right)$, then the $C Q$ algorithm converges to a solution of (1.1), whenever the solution set is nonempty. However, in order to actualize the $C Q$ algorithm, computing the operator norm of $A$ is a very complicated work in practice.

(c) The Author(s) 2020. This article is licensed under a Creative Commons Attribution 4.0 International License, which permits use, sharing, adaptation, distribution and reproduction in any medium or format, as long as you give appropriate credit to the original author(s) and the source, provide a link to the Creative Commons licence, and indicate if changes were made. The images or other third party material in this article are included in the article's Creative Commons licence, unless indicated otherwise in a credit line to the material. If material is not included in the article's Creative Commons licence and your intended use is not permitted by statutory regulation or exceeds the permitted use, you will need to obtain permission directly from the copyright holder. To view a copy of this licence, visit http://creativecommons.org/licenses/by/4.0/. 
As a prolongation of problem (1.1), the split common fixed point problem (SCFPP) has been extensively researched in recent years. The SCFPP is an inverse problem, which aims to find an element in a fixed point set so that the image under a bounded linear operator belongs to another fixed point set. More specifically, the SCFPP is looking for a $q \in B_{1}$ such that

$$
q \in F(U) \text { such that } A q \in F(T) \text {, }
$$

where $A: B_{1} \longrightarrow B_{2}$ is the bounded linear operator, and $U: B_{1} \longrightarrow B_{1}, T: B_{2} \longrightarrow B_{2}$ are the two nonlinear operators. We denote by $F(U)$ and $F(T)$ the sets of fixed points of $U$ and $T$, respectively. $\Delta$ denotes the set of solutions of SCFPP, that is,

$$
\Delta=\{q \in F(U): A q \in F(T)\}
$$

In particularly, if $T$ and $U$ are both the identity operator, then the SCFPP is clearly changed to the SFP.

A typical method for solving the SCFPP is to use the following iterative algorithm:

$$
u_{n+1}=U\left(I-\delta A^{*}(I-T) A\right) u_{n}, \quad n \geq 0 .
$$

It is shown in [7] that, if the stepsize $\delta \in\left(0, \frac{2}{\|A\|^{2}}\right)$ and the operators in (1.3) are directed, then the sequence generated by algorithm (1.4) converges weakly to a solution of the SCFPP whenever such a solution exists.

Moudafi [8] introduced an iteration scheme for demicontractive mappings and obtained a weak convergence theorem for the SCFPP in Hilbert spaces. Since then, many authors have studied the SCFPP of other mappings in the frameworks of two Hilbert spaces (see, for instance, [9-13])

In 2015, Tang et al. [14] obtained a weak convergence theorem of the SCFPP for the asymptotically nonexpansive mapping $S$ in Banach spaces of the following algorithm:

$$
\left\{\begin{array}{l}
z_{n}=u_{n}+\delta J_{1}^{-1} A^{*} J_{2}(T-I) A u_{n}, \\
u_{n+1}=\left(1-\alpha_{n}\right) z_{n}+\alpha_{n} S^{n} z_{n} .
\end{array}\right.
$$

They showed that the sequence $\left\{u_{n}\right\}$ generated by (1.5) converges weakly to a $q \in \Delta$.

Recently, Tang et al. [15] studied and proved a strong convergence theorem for the SCFPP (1.3) in infinite dimensional real Hilbert spaces based on the viscosity approximation, a single-step regularized method working as follows:

$$
u_{n+1}=\alpha_{n} u_{n}+\beta_{n} h\left(u_{n}\right)+\gamma_{n} S\left(I-\xi_{n} A^{*}(I-T) A\right) u_{n}, \quad n \geq 0,
$$

where $S$ and $T$ are firmly nonexpansive mappings for which both $I-S$ and $I-T$ are demiclosed at zero, and $h: H \longrightarrow H$ is an $\alpha$-contraction mapping with $\alpha \in(0,1)$.

In this article, inspired by the above results, we consider and study the SCFPP for asymptotically nonexpansive mappings in the frameworks of two real Banach spaces. That is, we present an iterative algorithm to approximate a solution of the SCFPP and show some 
strong convergence theorems under appropriate conditions, which also solve some variational inequalities. Therefore, we extend the main results of Tang et al. [14] and Hong et al. [16] from Hilbert spaces to Banach spaces and from firmly nonexpensive mappings to asymptotically nonexpansive mappings. In some cases, some other results are also improved (see $[8,15,17,18])$.

\section{Preliminaries}

The following are some definitions and lemmas that will be used in the proof of the main results in the next section.

Throughout this paper, let $B$ be a real Banach space and $B^{*}$ be the dual space of $B$. The normalized duality mapping $J: B \rightarrow 2^{B^{*}}$ is defined by

$$
J(x)=\left\{f \in B^{*}:\langle x, f\rangle=\|x\|\|f\|,\|x\|=\|f\|\right\}, \quad \forall x \in B,
$$

where $\langle.,$.$\rangle denotes the duality pairing. As is well known (see e.g. [6]), the operator J$ is well defined and $J$ is multiple-valued and nonlinear in general. And $J$ is an identity mapping if and only if $B$ is a Hilbert space.

A Banach space $B$ is said to be strictly convex if $\frac{\|u+v\|}{2}<1$ for $\|u\|=\|v\|=1$ and $u \neq v$. The modulus of convexity of $B$ is defined by

$$
\delta_{B}(\epsilon)=\inf \left\{1-\left\|\frac{1}{2}(u+v)\right\|:\|u\|,\|v\| \leq 1,\|u-v\| \geq \epsilon\right\},
$$

for all $0 \leq \epsilon \leq 2$. $B$ is called uniformly convex, if for all $0<\epsilon \leq 2$ such that $\delta_{B}(0)=0$ and $\delta_{B}(\epsilon)>0$.

Let $\rho_{B}:[0,+\infty) \longrightarrow[0,+\infty)$ be the modulus of smoothness of $B$ which is defined by

$$
\rho_{B}(s)=\sup \left\{\frac{1}{2}(\|u+v\|+\|u-v\|)-1:\|u\|=1,\|v\| \leq s\right\} .
$$

A Banach space $B$ is called uniformly smooth if $\frac{\rho_{B}(s)}{s} \rightarrow 0$ as $s \rightarrow 0$. Then a Banach space $B$ is called $q$-uniformly smooth, if, for all $s>0$, there exists a constant $c>0$ such that $\rho_{B}(s) \geq c s^{q}$. It is known that every $q$-uniformly smooth Banach space is uniformly smooth.

Definition 2.1 Let $C$ be a nonempty closed convex subset of a Banach space $B$ and $T$ : $C \rightarrow C$ be a mapping, then

- $T$ is called a contraction if there exists a constant $k \in(0,1)$ satisfying

$$
\|T(u)-T(v)\| \leq k\|u-v\|, \quad \forall u, v \in C .
$$

- $T$ is called a nonexpansive mapping if the above inequality is also true for $k=1$.

- $T$ is called a firmly nonexpansive mapping if

$$
\|T u-T v\|^{2} \leq\langle T u-T v, u-v\rangle .
$$

- $T$ is called an asymptotically nonexpansive mapping, if, for all $u, v \in C$, there exists a sequence $\left\{k_{n}\right\}$ with $\lim _{n \rightarrow \infty} k_{n}=1$ such that

$$
\left\|T^{n} u-T^{n} v\right\| \leq k_{n}\|u-v\| .
$$


It is easy to see that every nonexpansive mapping is an asymptotically nonexpansive mapping.

Definition 2.2 Let $C$ be a nonempty closed convex subset of a real Banach space $B$, mapping $U: C \longrightarrow B$ is said to be uniformly regular if

$$
\lim _{n \rightarrow \infty} \sup _{u \in C}\left\|U^{n+1} u-U^{n} u\right\|=0
$$

Lemma 2.1 ([19]) If B is a 2-uniformly smooth Banach space with the best smoothness constant $t>0$, we have the relation

$$
\|u+v\|^{2} \leq\|u\|^{2}+2\langle v, J u\rangle+2\|t v\|^{2}
$$

Lemma 2.2 ([20]) Let $\left\{w_{n}\right\}$ and $\left\{z_{n}\right\}$ be bounded sequences in a Banach space $B$ and $\left\{\alpha_{n}\right\}$ be a sequence in $[0,1]$ with $0<\liminf _{n \rightarrow \infty} \alpha_{n} \leq \limsup _{n \rightarrow \infty} \alpha_{n}<1$. Suppose that $w_{n+1}=\left(1-\alpha_{n}\right) w_{n}+\alpha_{n} z_{n}$ for all $n \geq 0$ and $\limsup _{n \rightarrow \infty}\left(\left\|z_{n+1}-z_{n}\right\|-\left\|w_{n+1}-w_{n}\right\|\right) \leq 0$. Then $\lim _{n \rightarrow \infty}\left\|z_{n}-w_{n}\right\|=0$.

Lemma 2.3 ([21]) Let $C$ be a nonempty bounded and closed convex subset of a reflexive smooth Banach space $B$ and $J$ be a weakly sequential continuous normal duality mapping $T: C \rightarrow C$ be an asymptotical nonexpansive mapping. Then $I-T$ is demiclosed at zero, i.e., if $u_{n} \rightarrow u$ weakly and $u_{n}-T u_{n} \rightarrow 0$ strongly, then $u \in F(T)$.

Lemma 2.4 ([22]) Assume $\left\{p_{n}\right\}$ is a sequence of nonnegative real numbers such that

$$
p_{n+1} \leq\left(1-\sigma_{n}\right) p_{n}+\xi_{n}, \quad n \geq 0,
$$

where $\left\{\sigma_{n}\right\}$ is a sequence in $(0,1)$ and $\left\{\xi_{n}\right\}$ is a real sequence such that

$$
\begin{aligned}
& \text { (1) } \lim _{n \rightarrow \infty} \sigma_{n}=0 \text { and } \sum_{n=0}^{\infty} \sigma_{n}=\infty \text {; } \\
& \text { (2) } \limsup _{n \rightarrow \infty} \frac{\xi_{n}}{\sigma_{n}} \leq 0 \quad \text { or } \quad \sum_{n=1}^{\infty}\left|\xi_{n}\right|<\infty \text {. }
\end{aligned}
$$

Then $\lim _{n \rightarrow \infty} p_{n}=0$.

\section{Main results}

Theorem 3.1 Let $B_{1}$ be a real strictly convex and 2-uniformly smooth Banach space with the best smoothness constant $t$ satisfying $0<t<\frac{1}{\sqrt{2}}$ and a weakly sequential continuous normal duality mapping J, $B_{2}$ be a real smooth Banach space. Suppose that $h: B_{1} \longrightarrow B_{1}$ is a contraction mapping with contractive coefficient $k \in(0,1)$ and $A: B_{1} \longrightarrow B_{2}$ is a bounded linear operator and $A^{*}$ is the adjoint of $A$. Let $T: B_{2} \longrightarrow B_{2}$ be a nonexpansive mapping and $U: B_{1} \longrightarrow B_{1}$ be an asymptotically nonexpansive mapping with asymptotical coefficient sequence $\left\{k_{n}\right\}$ and $F(U) \neq \emptyset$. Assume that the SCFPP (1.3) has a nonempty solution set $\Delta$ 
and $U$ is uniformly regular in $\Delta$. Let $\left\{u_{n}\right\}$ be a sequence generated by

$$
\left\{\begin{array}{l}
u_{1} \in B_{1}, \\
v_{n}=u_{n}-\delta J_{B_{1}}^{-1} A^{*} J_{B_{2}}(I-T) A u_{n}, \\
u_{n+1}=\alpha_{n} u_{n}+\gamma_{n} h\left(u_{n}\right)+\zeta_{n} U^{n} v_{n},
\end{array}\right.
$$

where $\left\{\alpha_{n}\right\},\left\{\gamma_{n}\right\},\left\{\zeta_{n}\right\} \subset(0,1)$, satisfying the following conditions:

(i) $\quad \alpha_{n}+\gamma_{n}+\zeta_{n}=1, \quad 0<\liminf _{n \rightarrow \infty} \alpha_{n} \leq \limsup _{n \rightarrow \infty} \alpha_{n}<1$;

(ii) $\quad \lim _{n \rightarrow \infty} \gamma_{n}=0, \quad \sum_{n=0}^{\infty} \gamma_{n}=\infty, \quad \eta \gamma_{n}=k_{n}-1, \quad 0<\eta<1-k$;

(iii) $\delta \in\left(0, \frac{1-2 t^{2}}{\|A\|^{2}}\right)$.

Then the sequence $\left\{u_{n}\right\}$ generated by (3.1) converges strongly to a point $q=P_{\Delta} h(q) \in \Delta$, which also solves the variational inequality:

$$
\langle(I-h) q, j(q-w)\rangle \leq 0, \quad \forall w \in \Delta .
$$

Proof Since $P_{\Delta} h$ is a contraction on $B_{1}$, there exists an unique element $q \in B_{1}$ such that $q=P_{\Delta} h(q)$ by the Banach contraction principle. So there is a $q \in \Delta$. Now, we split the proof into five steps.

Step 1 First we show that the sequence $\left\{u_{n}\right\}$ is bounded. For any given $q \in \Delta$, it follows from (3.1), condition (iii) and Lemma 2.1 that

$$
\begin{aligned}
\left\|v_{n}-q\right\|^{2}= & \left\|\left(u_{n}-q\right)+\delta J_{B_{1}}^{-1} A^{*} J_{B_{2}}(T-I) A u_{n}\right\|^{2} \\
\leq & \delta^{2}\left\|J_{B_{1}}^{-1} A^{*} J_{B_{2}}(T-I) A u_{n}\right\|^{2}+2 \delta\left\langle u_{n}-q, A^{*} J_{B_{2}}(T-I) A u_{n}\right\rangle \\
& +2 t^{2}\left\|u_{n}-q\right\|^{2} \\
\leq & \delta^{2}\|A\|^{2}\left\|(T-I) A u_{n}\right\|^{2}+2 t^{2}\left\|u_{n}-q\right\|^{2} \\
& +2 \delta\left\langle A u_{n}-A q, J_{B_{2}}(T-I) A u_{n}\right\rangle \\
= & \delta^{2}\|A\|^{2}\left\|(T-I) A u_{n}\right\|^{2}+2 t^{2}\left\|u_{n}-q\right\|^{2}-2 \delta\left\|(T-I) A u_{n}\right\|^{2} \\
& +2 \delta\left\langle T A u_{n}-A q, J_{B_{2}}(T-I) A u_{n}\right\rangle \\
\leq & \left(\delta^{2}\|A\|^{2}-2 \delta\right)\left\|(T-I) A u_{n}\right\|^{2}+2 t^{2}\left\|u_{n}-q\right\|^{2} \\
& +\delta\left(\left\|T A u_{n}-A q\right\|^{2}+\left\|(T-I) A u_{n}\right\|^{2}\right) \\
\leq & 2 t^{2}\left\|u_{n}-q\right\|^{2}+\left(\delta^{2}\|A\|^{2}-\delta\right)\left\|(T-I) A u_{n}\right\|^{2}+\delta\left\|A u_{n}-A q\right\|^{2} \\
\leq & \left(2 t^{2}+\delta\|A\|^{2}\right)\left\|u_{n}-q\right\|^{2}-\delta\left(1-\delta\|A\|^{2}\right)\left\|(T-I) A u_{n}\right\|^{2} \\
\leq & \left\|u_{n}-q\right\|^{2} .
\end{aligned}
$$

Because $0 \leq k<1$, by (3.1), (3.2) and condition (ii), we have

$$
\left\|u_{n+1}-q\right\|=\left\|\alpha_{n} u_{n}+\gamma_{n} h\left(u_{n}\right)+\zeta_{n} U^{n} v_{n}-q\right\|
$$




$$
\begin{aligned}
& =\| \alpha_{n}\left(u_{n}-q\right)+\gamma_{n}\left(h\left(u_{n}\right)-h(q)+\gamma_{n}(h(q)-q)+\zeta_{n}\left(U^{n} v_{n}-q\right) \|\right. \\
& \leq \alpha_{n}\left\|u_{n}-q\right\|+k \gamma_{n}\left\|u_{n}-q\right\|+\gamma_{n}\|h(q)-q\|+k_{n} \zeta_{n}\left\|v_{n}-q\right\| \\
& \leq\left(\alpha_{n}+k \gamma_{n}+k_{n} \zeta_{n}\right)\left\|u_{n}-q\right\|+\gamma_{n}\|h(q)-q\| \\
& =\left(1-\left(\gamma_{n}(1-k)-\zeta_{n}\left(k_{n}-1\right)\right)\right)\left\|u_{n}-q\right\|+\gamma_{n}\|h(q)-q\| \\
& \leq\left(1-\left(\gamma_{n}(1-k)-\eta \gamma_{n}\right)\right)\left\|u_{n}-q\right\|+\gamma_{n}\|h(q)-q\| \\
& =\left(1-\gamma_{n}(1-k-\eta)\right)\left\|u_{n}-q\right\|+\gamma_{n}(1-k-\eta) \frac{\|h(q)-q\|}{1-k-\eta} \\
& \leq \max \left\{\left\|u_{n}-q\right\|, \frac{\|h(q)-q\|}{1-k-\eta}\right\} .
\end{aligned}
$$

By induction, we readily obtain

$$
\left\|u_{n+1}-q\right\| \leq \max \left\{\left\|u_{0}-q\right\|, \frac{\|h(q)-q\|}{1-k-\eta}\right\} .
$$

This implies that $\left\{u_{n}\right\}$ is bounded, and so are $\left\{v_{n}\right\},\left\{h\left(u_{n}\right)\right\},\left\{U^{n} v_{n}\right\}$.

Step 2 We show that $\lim _{n \rightarrow \infty}\left\|u_{n+1}-u_{n}\right\|=0$ and $\lim _{n \rightarrow \infty}\left\|v_{n+1}-v_{n}\right\|=0$. To see this, we set $z_{n}=\frac{u_{n+1}-\alpha_{n} u_{n}}{1-\alpha_{n}}, \forall n \geq 0$. We have

$$
\begin{aligned}
z_{n+1}-z_{n}= & \frac{u_{n+2}-\alpha_{n+1} u_{n+1}}{1-\alpha_{n+1}}-\frac{u_{n+1}-\alpha_{n} u_{n}}{1-\alpha_{n}} \\
= & \frac{\gamma_{n+1} h\left(u_{n+1}\right)+\zeta_{n+1} U^{n+1} v_{n+1}}{1-\alpha_{n+1}}-\frac{\gamma_{n} h\left(u_{n}\right)+\zeta_{n} U^{n} v_{n}}{1-\alpha_{n}} \\
= & \frac{\gamma_{n+1} h\left(u_{n+1}\right)+\left(1-\alpha_{n+1}-\gamma_{n+1}\right) U^{n+1} v_{n+1}}{1-\alpha_{n+1}}-\frac{\gamma_{n} h\left(u_{n}\right)+\left(1-\alpha_{n}-\gamma_{n}\right) U^{n} v_{n}}{1-\alpha_{n}} \\
= & \frac{\gamma_{n+1}}{1-\alpha_{n+1}}\left[h\left(u_{n+1}\right)-h\left(u_{n}\right)\right]+\left(\frac{\gamma_{n+1}}{1-\alpha_{n+1}}-\frac{\gamma_{n}}{1-\alpha_{n}}\right) h\left(u_{n}\right) \\
& -\left(\frac{\gamma_{n+1}}{1-\alpha_{n+1}}-\frac{\gamma_{n}}{1-\alpha_{n}}\right) U^{n} v_{n}+\left(1-\frac{\gamma_{n+1}}{1-\alpha_{n+1}}\right)\left(U^{n+1} v_{n+1}-U^{n} v_{n}\right) \\
= & \frac{\gamma_{n+1}}{1-\alpha_{n+1}}\left[h\left(u_{n+1}\right)-h\left(u_{n}\right)\right]+\left(\frac{\gamma_{n+1}}{1-\alpha_{n+1}}-\frac{\gamma_{n}}{1-\alpha_{n}}\right)\left(h\left(u_{n}\right)-U^{n} v_{n}\right) \\
& +\left(1-\frac{\gamma_{n+1}}{1-\alpha_{n+1}}\right)\left(U^{n+1} v_{n+1}-U^{n+1} v_{n}\right)+\left(1-\frac{\gamma_{n+1}}{1-\alpha_{n+1}}\right)\left(U^{n+1} v_{n}-U^{n} v_{n}\right) .
\end{aligned}
$$

This implies that

$$
\begin{aligned}
\left\|z_{n+1}-z_{n}\right\| \leq & \frac{k \gamma_{n+1}}{1-\alpha_{n+1}}\left\|u_{n+1}-u_{n}\right\|+\left|\frac{\gamma_{n+1}}{1-\alpha_{n+1}}-\frac{\gamma_{n}}{1-\alpha_{n}}\right|\left\|h\left(u_{n}\right)-U^{n} v_{n}\right\| \\
& +\sup _{v \in \Delta}\left\|U^{n+1} v-U^{n} v\right\|+k_{n+1}\left(1-\frac{\gamma_{n+1}}{1-\alpha_{n+1}}\right)\left\|v_{n+1}-v_{n}\right\| .
\end{aligned}
$$

By Lemma 2.1 and condition (iii), we can get

$$
\begin{aligned}
\left\|v_{n+1}-v_{n}\right\|^{2} & =\left\|\left(u_{n+1}-u_{n}\right)-\left(\delta J_{B_{1}}^{-1} A^{*} J_{B_{2}}(I-T) A u_{n+1}-\delta J_{B_{1}}^{-1} A^{*} J_{B_{2}}(I-T) A u_{n}\right)\right\|^{2} \\
& \leq \delta^{2}\|A\|^{2}\left\|(I-T) A u_{n+1}-(I-T) A u_{n}\right\|^{2}+2 \delta^{2}\left\|u_{n+1}-u_{n}\right\|^{2}
\end{aligned}
$$




$$
\begin{aligned}
& -2 \delta\left\langle A u_{n+1}-A u_{n}, J_{B_{2}}(I-T) A u_{n+1}-J_{B_{2}}(I-T) A u_{n}\right\rangle \\
= & \delta^{2}\|A\|^{2}\left\|(I-T) A u_{n+1}-(I-T) A u_{n}\right\|^{2}+2 \delta^{2}\left\|u_{n+1}-u_{n}\right\|^{2} \\
& -2 \delta\left\|(I-T) A u_{n+1}-(I-T) A u_{n}\right\|^{2} \\
& +2 \delta\left\langle T A u_{n+1}-T A u_{n}, J_{B_{2}}(I-T) A u_{n+1}-J_{B_{2}}(I-T) A u_{n}\right\rangle \\
\leq & \left(\delta^{2}\|A\|^{2}-2 \delta\right)\left\|(I-T) A u_{n+1}-(I-T) A u_{n}\right\|^{2}+2 \delta^{2}\left\|u_{n+1}-u_{n}\right\|^{2} \\
& +\delta\left(\left\|T A u_{n+1}-T A u_{n}\right\|^{2}+\left\|(I-T) A u_{n+1}-(I-T) A u_{n}\right\|^{2}\right) \\
\leq & \left(\delta^{2}\|A\|^{2}-\delta\right)\left\|(I-T) A u_{n+1}-(I-T) A u_{n}\right\|^{2}+2 \delta^{2}\left\|u_{n+1}-u_{n}\right\|^{2} \\
& +\delta\|A\|^{2}\left\|u_{n+1}-u_{n}\right\|^{2} \\
= & \left(2 \delta^{2}+\delta\|A\|^{2}\right)\left\|u_{n+1}-u_{n}\right\|^{2}-\delta\left(1-\delta\|A\|^{2}\right)\left\|(I-T)\left(A u_{n+1}-A u_{n}\right)\right\|^{2} \\
\leq & \left\|u_{n+1}-u_{n}\right\|^{2} .
\end{aligned}
$$

Thus, we have $\left\|y_{n+1}-y_{n}\right\| \leq\left\|x_{n+1}-x_{n}\right\|$. Then, from (3.4), (3.5) and condition (ii), it follows that

$$
\begin{aligned}
\left\|z_{n+1}-z_{n}\right\| \leq & \left(\frac{k \gamma_{n+1}}{1-\alpha_{n+1}}+k_{n+1}\left(1-\frac{\gamma_{n+1}}{1-\alpha_{n+1}}\right)\right)\left\|u_{n+1}-u_{n}\right\| \\
& +\left|\frac{\gamma_{n+1}}{1-\alpha_{n+1}}-\frac{\gamma_{n}}{1-\alpha_{n}}\right|\left\|h\left(u_{n}\right)-U^{n} v_{n}\right\|+\sup _{v \in \Delta}\left\|U^{n+1} v-U^{n} v\right\| \\
= & \frac{k \gamma_{n+1}+k_{n+1} \zeta_{n+1}}{1-\alpha_{n+1}}\left\|u_{n+1}-u_{n}\right\| \\
& +\left|\frac{\gamma_{n+1}}{1-\alpha_{n+1}}-\frac{\gamma_{n}}{1-\alpha_{n}}\right|\left\|h\left(u_{n}\right)-U^{n} v_{n}\right\|+\sup _{v \in \Delta}\left\|U^{n+1} v-U^{n} v\right\| \\
\leq & \left(1-\frac{(1-k-\eta) \gamma_{n+1}}{1-\alpha_{n+1}}\right)\left\|u_{n+1}-u_{n}\right\| \\
& +\left|\frac{\gamma_{n+1}}{1-\alpha_{n+1}}-\frac{\gamma_{n}}{1-\alpha_{n}}\right|\left\|h\left(u_{n}\right)-U^{n} v_{n}\right\|+\sup _{v \in \Delta}\left\|U^{n+1} v-U^{n} v\right\| .
\end{aligned}
$$

Therefore, by condition (ii), we have

$$
\limsup _{n \rightarrow \infty}\left(\left\|z_{n+1}-z_{n}\right\|-\left\|u_{n+1}-u_{n}\right\|\right) \leq 0 .
$$

It follows form Lemma 2.2 and condition $(i)$ that

$$
\lim _{n \rightarrow \infty}\left\|z_{n}-u_{n}\right\|=0 .
$$

Note that $z_{n}=\frac{u_{n+1}-\alpha_{n} u_{n}}{1-\alpha_{n}}$, it is easy to see that

$$
\lim _{n \rightarrow \infty}\left\|u_{n+1}-u_{n}\right\|=0 .
$$

Clearly, from (3.5) we obtain

$$
\lim _{n \rightarrow \infty}\left\|v_{n+1}-v_{n}\right\|=0
$$


Step 3 We prove that $\left\|u_{n}-U u_{n}\right\| \rightarrow 0$, as $n \rightarrow \infty$. We have

$$
\begin{aligned}
\left\|u_{n+1}-U^{n} v_{n}\right\| & =\left\|U^{n} v_{n} u_{n}+\gamma_{n} h\left(u_{n}\right)-\alpha_{n} U^{n} v_{n}-\gamma_{n} U^{n} v_{n}\right\| \\
& =\left\|\alpha_{n}\left(u_{n}-U^{n} v_{n}\right)+\gamma_{n}\left(h\left(u_{n}\right)-U^{n} v_{n}\right)\right\| \\
& \leq \alpha_{n}\left\|u_{n}-u_{n+1}\right\|+\alpha_{n}\left\|u_{n+1}-U^{n} v_{n}\right\|+\gamma_{n}\left\|h\left(u_{n}\right)-U^{n} v_{n}\right\| .
\end{aligned}
$$

We can get

$$
\left\|u_{n+1}-U^{n} v_{n}\right\| \leq \frac{\alpha_{n}}{1-\alpha_{n}}\left\|u_{n+1}-u_{n}\right\|+\frac{\gamma_{n}}{1-\alpha_{n}}\left\|h\left(u_{n}\right)-U^{n} v_{n}\right\| .
$$

By (3.6) and condition (ii), we have

$$
\lim _{n \rightarrow \infty}\left\|u_{n+1}-U^{n} v_{n}\right\|=0
$$

By (3.2) and condition (iii), we obtain

$$
\begin{aligned}
\left\|u_{n+1}-q\right\|^{2}= & \left\|\alpha_{n} u_{n}+\gamma_{n} h\left(u_{n}\right)+\zeta_{n} U^{n} v_{n}-q\right\|^{2} \\
\leq & \alpha_{n}\left\|u_{n}-q\right\|^{2}+\gamma_{n}\left\|h\left(u_{n}\right)-q\right\|^{2}+\zeta_{n}\left\|U^{n} v_{n}-q\right\|^{2} \\
\leq & \alpha_{n}\left\|u_{n}-q\right\|^{2}+\gamma_{n}\left\|h\left(u_{n}\right)-q\right\|^{2}+\zeta_{n} k_{n}^{2}\left\|v_{n}-q\right\|^{2} \\
\leq & \alpha_{n}\left\|u_{n}-q\right\|^{2}+\gamma_{n}\left\|h\left(u_{n}\right)-q\right\|^{2} \\
& +\zeta_{n} k_{n}^{2}\left[\left(2 t^{2}+\delta\|A\|^{2}\right)\left\|u_{n}-q\right\|^{2}-\delta\left(1-\delta\|A\|^{2}\right)\left\|(T-I) A u_{n}\right\|^{2}\right] \\
\leq & \left(\alpha_{n}+\zeta_{n} k_{n}^{2}\right)\left\|u_{n}-q\right\|^{2}+\gamma_{n}\left\|h\left(u_{n}\right)-q\right\|^{2} \\
& -\zeta_{n} k_{n}^{2} \delta\left(1-\delta\|A\|^{2}\right)\left\|T A u_{n}-A u_{n}\right\|^{2} .
\end{aligned}
$$

By the last inequality and condition (i) we can get

$$
\begin{aligned}
\left\|T A u_{n}-A u_{n}\right\|^{2} \leq & \frac{\left(\alpha_{n}+\zeta_{n} k_{n}^{2}-1\right)\left\|u_{n}-q\right\|^{2}+\gamma_{n}\left\|h\left(u_{n}\right)-q\right\|^{2}}{\zeta_{n} k_{n}^{2} \delta\left(1-\delta\|A\|^{2}\right)} \\
& +\frac{\left(\left\|u_{n}-q\right\|+\left\|u_{n+1}-q\right\|\right)\left\|u_{n+1}-u_{n}\right\|}{\zeta_{n} k_{n}^{2} \delta\left(1-\delta\|A\|^{2}\right)} \\
= & \frac{\left(\zeta_{n}\left(k_{n}^{2}-1\right)-\gamma_{n}\right)\left\|u_{n}-q\right\|^{2}+\gamma_{n}\left\|h\left(u_{n}\right)-q\right\|^{2}}{\zeta_{n} k_{n}^{2} \delta\left(1-\delta\|A\|^{2}\right)} \\
& +\frac{\left(\left\|u_{n}-q\right\|+\left\|u_{n+1}-q\right\|\right)\left\|u_{n+1}-u_{n}\right\|}{\zeta_{n} k_{n}^{2} \delta\left(1-\delta\|A\|^{2}\right)} .
\end{aligned}
$$

By condition (ii) and applying Step 2, we have

$$
\lim _{n \rightarrow \infty}\left\|T A u_{n}-A u_{n}\right\|=0
$$

Considering the bounded sequence $\left\{u_{n}\right\}$, it must have a convergent subsequence $\left\{u_{n_{k}}\right\}$. There exists a subsequence $\left\{u_{n_{k_{j}}}\right\}$ of $\left\{u_{n_{k}}\right\}$ such that $u_{n_{k_{j}}} \rightarrow w \in B_{1}$. Without loss of generality, we assume that $u_{n_{k}} \rightarrow w$ as $k \rightarrow \infty$. Therefore, $A u_{n_{k}} \rightarrow A w$ as $k \rightarrow \infty$ and

$$
\lim _{n \rightarrow \infty}\left\|T A u_{n_{k}}-A u_{n_{k}}\right\|=0
$$


Since $v_{n}=u_{n}-\delta J_{B_{1}}^{-1} A^{*} J_{B_{2}}(I-T) A u_{n},\left\|u_{n}-v_{n}\right\|=\left\|\delta J_{B_{1}}^{-1} A^{*} J_{B_{2}}(I-T) A u_{n}\right\|$, we can get

$$
\lim _{n \rightarrow \infty}\left\|u_{n}-v_{n}\right\|=0
$$

Moreover, we have

$$
\begin{aligned}
\left\|u_{n}-U^{n} u_{n}\right\| & \leq\left\|u_{n}-u_{n+1}\right\|+\left\|u_{n+1}-U^{n} v_{n}\right\|+\left\|U^{n} v_{n}-U^{n} u_{n}\right\| \\
& \leq\left\|u_{n+1}-u_{n}\right\|+\left\|u_{n+1}-U^{n} v_{n}\right\|+k_{n}\left\|v_{n}-u_{n}\right\|
\end{aligned}
$$

In view of Step 2 and (3.7), (3.9), we obtain

$$
\lim _{n \rightarrow \infty}\left\|u_{n}-U^{n} u_{n}\right\|=0
$$

Furthermore, we have

$$
\begin{aligned}
\left\|u_{n}-U u_{n}\right\|= & \left\|u_{n}-u_{n+1}+u_{n+1}-U^{n+1} u_{n+1}+U^{n+1} u_{n+1}-U^{n+1} u_{n} U^{n+1} u_{n}-U u_{n}\right\| \\
\leq & \left\|u_{n+1}-u_{n}\right\|+\left\|u_{n+1}-U^{n+1} u_{n+1}\right\|+\left\|U^{n+1} u_{n+1}-U^{n+1} u_{n}\right\| \\
& +\left\|U^{n+1} u_{n}-U u_{n}\right\| \\
\leq & \left\|u_{n+1}-u_{n}\right\|+\left\|u_{n+1}-U^{n+1} u_{n+1}\right\|+k_{n+1}\left\|u_{n+1}-u_{n}\right\|+k_{1}\left\|U^{n} u_{n}-u_{n}\right\| \\
= & \left(1+k_{n+1}\right)\left\|u_{n+1}-u_{n}\right\|+\left\|u_{n+1}-U^{n+1} u_{n+1}\right\|+k_{1}\left\|U^{n} u_{n}-u_{n}\right\| .
\end{aligned}
$$

By (3.6) and (3.10), we can get

$$
\lim _{n \rightarrow \infty}\left\|u_{n}-U u_{n}\right\|=0
$$

Step 4 Since $B_{1}$ is a reflexive Banach space and $\left\{u_{n}\right\}$ is bounded, there exists a subsequence $u_{n_{k}} \rightarrow w \in B_{1}$ as $n \rightarrow \infty$. And

$$
\lim _{n \rightarrow \infty}\left\langle(I-h) q, j\left(q-u_{n_{k}}\right)\right\rangle=\limsup _{n \rightarrow \infty}\left\langle(I-h) q, j\left(q-u_{n}\right)\right\rangle
$$

By Step 3, we know $A u_{n} \rightarrow A w$. Because $B_{1}$ and $B_{2}$ are reflexive smooth Banach spaces, it follows from Step 3 and Lemma 2.3, that $A w \in F(T)$. That is, $w \in \Delta$.

On the other hand, since $q \in \Delta$ satisfies

$$
\langle(I-h) q, j(q-w)\rangle \leq 0, \quad \forall w \in \Delta,
$$

and because $J$ is a weakly sequential continuous duality mapping, we obtain

$$
\begin{aligned}
\limsup _{n \rightarrow \infty}\left\langle(I-h) q, j\left(q-u_{n}\right)\right\rangle & =\lim _{k \rightarrow \infty}\left\langle(I-h) q, j\left(q-u_{n_{k}}\right)\right\rangle \\
& =\langle(I-h) q, j(q-w)\rangle \leq 0 .
\end{aligned}
$$


Step 5 Finally, we prove that $\left\{u_{n}\right\}$ converges strongly to $q \in \Delta$. We have

$$
\begin{aligned}
\left\|u_{n+1}-q\right\|^{2}= & \left\langle\alpha_{n} u_{n}+\gamma_{n} h\left(u_{n}\right)+\zeta_{n} U^{n} v_{n}-q, j\left(u_{n+1}-q\right)\right\rangle \\
= & \alpha_{n}\left\langle u_{n}-q, j\left(u_{n+1}-q\right)\right\rangle+\gamma_{n}\left\langle h\left(u_{n}\right)-q, j\left(u_{n+1}-q\right)\right\rangle \\
& +\zeta_{n}\left\langle U^{n} v_{n}-q, j\left(u_{n+1}-q\right)\right\rangle \\
\leq & \alpha_{n}\left\langle u_{n}-q, j\left(u_{n+1}-q\right)\right\rangle+\gamma_{n}\left\langle h\left(u_{n}\right)-h(q), j\left(u_{n+1}-q\right)\right\rangle \\
& +\gamma_{n}\left\langle h(q)-q, j\left(u_{n+1}-q\right)\right\rangle+\zeta_{n}\left\langle U^{n} v_{n}-q, j\left(u_{n+1}-q\right)\right\rangle \\
\leq & \alpha_{n}\left\|u_{n}-q\right\|\left\|u_{n+1}-q\right\|+k \gamma_{n}\left\|u_{n}-q\right\|\left\|u_{n+1}-q\right\| \\
& +\gamma_{n}\left\langle h(q)-q, j\left(u_{n+1}-q\right)\right\rangle+k_{n} \zeta_{n}\left\|v_{n}-q\right\|\left\|u_{n+1}-q\right\| \\
\leq & \left(\alpha_{n}+k \gamma_{n}+k_{n} \zeta_{n}\right)\left\|u_{n}-q\right\|\left\|u_{n+1}-q\right\|+\gamma_{n}\left\langle h(q)-q, j\left(u_{n+1}-q\right)\right\rangle \\
\leq & \frac{\alpha_{n}+k \gamma_{n}+k_{n} \zeta_{n}}{2}\left\|u_{n}-q\right\|^{2}+\frac{\alpha_{n}+k \gamma_{n}+k_{n} \zeta_{n}}{2}\left\|u_{n+1}-q\right\|^{2} \\
& +\gamma_{n}\left\langle h(q)-q, j\left(u_{n+1}-q\right)\right\rangle .
\end{aligned}
$$

This implies that

$$
\begin{aligned}
\left(1-\frac{\alpha_{n}+k \gamma_{n}+k_{n} \zeta_{n}}{2}\right)\left\|u_{n+1}-q\right\|^{2} \leq & \frac{\alpha_{n}+k \gamma_{n}+k_{n} \zeta_{n}}{2}\left\|u_{n}-q\right\|^{2} \\
& +\gamma_{n}\left\langle h(q)-q, j\left(u_{n+1}-q\right)\right\rangle .
\end{aligned}
$$

That is,

$$
\begin{aligned}
\left\|u_{n+1}-q\right\|^{2} \leq & \frac{\alpha_{n}+k \gamma_{n}+k_{n} \zeta_{n}}{2-\left(\alpha_{n}+k \gamma_{n}+k_{n} \zeta_{n}\right)}\left\|u_{n}-q\right\|^{2} \\
& +\frac{2 \gamma_{n}}{2-\left(\alpha_{n}+k \gamma_{n}+k_{n} \zeta_{n}\right)}\left\langle h(q)-q, j\left(u_{n+1}-q\right)\right\rangle \\
\leq & \left(1-\frac{2 \gamma_{n}(1-k-\eta)}{2-\left(\alpha_{n}+k \gamma_{n}+k_{n} \zeta_{n}\right)}\right)\left\|u_{n}-q\right\|^{2} \\
& +\frac{2 \gamma_{n}}{2-\left(\alpha_{n}+k \gamma_{n}+k_{n} \zeta_{n}\right)}\left\langle h(q)-q, j\left(u_{n+1}-q\right)\right\rangle .
\end{aligned}
$$

Let

$$
\sigma_{n}=\frac{2 \gamma_{n}(1-k-\eta)}{2-\left(\alpha_{n}+k \gamma_{n}+k_{n} \zeta_{n}\right)}, \quad \xi_{n}=\frac{2 \gamma_{n}}{2-\left(\alpha_{n}+k \gamma_{n}+k_{n} \zeta_{n}\right)}\left\langle h(q)-q, j\left(u_{n+1}-q\right)\right\rangle .
$$

By conditions $(i)$ and $(i i)$, we know that

$$
\lim _{n \rightarrow \infty} \sigma_{n}=0 \quad \text { and } \quad \sigma_{n}=\frac{2 \gamma_{n}(1-k-\eta)}{2-\left(\alpha_{n}+k \gamma_{n}+k_{n} \zeta_{n}\right)} \geq \gamma_{n}(1-k-\eta) .
$$

Because $\sum_{n=0}^{\infty} \gamma_{n}=\infty, \sum_{n=0}^{\infty} \sigma_{n}=\infty$. In addition, by (3.12) we have

$$
\limsup _{n \rightarrow \infty} \frac{\xi_{n}}{\sigma_{n}}=\limsup _{n \rightarrow \infty} \frac{\left\langle h(q)-q, j\left(u_{n+1}-q\right)\right\rangle}{1-k-\eta} \leq 0 .
$$


Thus, applying Lemma 2.4 and (3.13), we conclude that

$$
\lim _{n \rightarrow \infty}\left\|u_{n}-q\right\|=0
$$

This completes the proof.

Remark 3.1 We know that each firmly nonexpansive mapping is a nonexpansive mapping and every nonexpansive mapping is an asymptotically nonexpansive mapping. In this paper, we research the SCFPP for asymptotically nonexpansive mappings in 2-uniformly Banach space. So there are five features to explain in detail:

1 If $\gamma_{n}=0$ in Theorem 3.1, then $\left\{u_{n}\right\}$ converges strongly to a fixed point of $U$. It is the main result of Tang et al. [14].

2 Since Hilbert space, $L^{p}(1<p \leq 2)$ space, etc. are 2-uniformly convex spaces, if $U$ is a firmly nonexpansive mapping and $B_{1}, B_{2}$ are Hilbert spaces in Theorem 3.1, then we obtain the main results of Tang et al. [15].

3 If $g\left(u_{n}\right)=c, U$ is a firmly nonexpansive mapping and $B_{1}, B_{2}$ are Hilbert spaces in the iterative sequence $\left\{u_{n}\right\}$ in Theorem 3.1, then we obtain the main results of Hong et al. [16].

4. If $U$ is a nonexpansive mapping and $B_{1}$ is a Hilbert space in Theorem 3.1, then we obtain the main results of Tang et al. [17].

5 It is well known that a firmly nonexpansive mapping includes resolvents and projection operators. Let $C, D$ be nonempty closed convex subsets of $B_{1}, B_{2}$, respectively. When $U^{n}=P_{C}, T=P_{Q}$, then (3.1) can also solve the split feasibility problem. That is, our Theorem 3.1 generalizes and improves the main results of Deepho J and Kuman P [18].

\section{Acknowledgements}

The authors thank the National Natural Science Foundation of China (Grant no. 11671365) for partially support about this research. The authors would like to thank the referees for their esteemed comments and suggestions.

\section{Funding}

This work was supported by the National Natural Science Foundation of China (Grant no. 11671365).

\section{Abbreviations}

$F(T)$, the set of fixed points of a mapping $T ; F(U)$, the set of fixed points of a mapping $U ; \Delta$, the set of solutions of split common fixed point problem; SFP, split feasibility problem; SCFPP, split common fixed point problem.

\section{Availability of data and materials}

Not applicable.

\section{Competing interests}

The authors declare that they have no competing interests.

\section{Authors' contributions}

All authors contributed equally and significantly in this research work. All authors read and approved the final manuscript.

\section{Publisher's Note}

Springer Nature remains neutral with regard to jurisdictional claims in published maps and institutional affiliations.

Received: 13 October 2020 Accepted: 30 November 2020 Published online: 08 December 2020

\section{References}

1. Censor, Y., Elfving, T.: A multiprojection algorithm using Bregman projection in product space. Numer. Algorithms 8 , 221-239 (1994)

2. Byrne, C.: Iterative oblique projection onto convex sets and the split feasibility problem. Inverse Probl. 18, 441-453 (2002) 
3. Byrne, C.: A unified treatment of some iterative algorithms in signal processing and image reconstruction. Inverse Probl. 20, 103-120 (2004)

4. Censor, Y., Bortfeld, T., Martin, B., et al.: A unified approach for inversion problem in intensity-modulated radiation therapy. Phys. Med. Biol. 51(10), 2253-2365 (2006)

5. Censor, Y., Elfving, T., Kopf, N., et al.: The multiple-sets split feasibility problem and its applications for inverse problems. Inverse Probl. 21(6), 2071-2084 (2005)

6. Censor, Y., Motiva, A., Segal, A.: Perturbed projections an subgradient projections for the multiple-sets split feasibility problem. J. Math. Anal. Appl. 327(2), 1244-1256 (2007)

7. Censor, Y., Segal, A.: The split common fixed point problem for directed operators. J. Convex Anal. 16, 587-600 (2009)

8. Moudafi, A.: The split common fixed point problem for demi-contractive mappings. Inverse Probl. 2010, 26 (2010)

9. Chang, S.S., Wang, L., Tang, Y.K., Yang, L.: The split common fixed point problem for total asymptotically strictly pseudocontractive mappings. J. Appl. Math. 2012, Article ID 385638, 13 pp. (2012)

10. Yao, Y.H., Yao, J.C., Liou, Y.C., Mihai, P.: Iterative algorithms for split common fixed points of demicontractive operators without priori knowledge of operator norms. Carpath. J. Math. 34(3), 459-466 (2018)

11. Duan, P.C., Zheng, X.B., Zhao, J.: Strong convergence theorems of viscosity iterative algorithms for split common fixed point problems. Mathematics 7(1), 14 (2019)

12. Zhang, X.F., Wang, L., Ma, Z.L., Qin, L.J.: The strong convergence theorems for split common fixed point problem of asymptotically nonexpansive mappings in Hilbert spaces. J. Inequal. Appl. 2015, 1 (2015)

13. Shahrosvand, A.A.E., Azizi, A.: The split common fixed point problem for a family of multivalued quasinonexpansive mappings and totally asymptotically strictly pseudocontractive mappings in Banach spaces. Mathematics 5(1), 11 (2017)

14. Tang, J.F., Chang, S.S., Wang, L., Wang, X.R.: On the split common fixed point problem for strict pseudocontractive and asymptotically nonexpansive mappings in Banach spaces. J. Inequal. Appl. 2015, 305 (2015)

15. Tang, J.F., Chang, S.S., Liu, M.: General split feasibility problems for families of nonexpansive mappings in Hilbert spaces. Acta Math. Sci. 36B(2), 602-613 (2016)

16. Hong, C.C., Huang, Y.Y.: A strong convergence algorithm for the two-operator split common fixed point problem in Hilbert spaces. Abstr. Appl. Anal. 2014, Article ID 350479, 8 pages (2014)

17. Mann, T.W.: Halpern iterations for the split common fixed point problem in Banach spaces. Linear Nonlinear Anal. 3, $1-18(2017)$

18. Deepho, J., Kuman, P.: A viscosity approximation method for the split feasibility problems. Trans. Eng. Technol. 2(6), 69-77 (2014)

19. Qin, X.L., Yao, J.C.: Weak convergence of a Mann-like algorithm for nonexpansive and accretive operators. J. Inequal. Appl. 2016, Article ID 232, 1-9 (2016)

20. Wang, Y.H., Pan, C.J.: The modified viscosity implicit rules for uniformly L-Lipschitzian asymptotically pseudocontractive mappings in Banach spaces. J. Nonlinear Sci. Appl. 10, 1582-1592 (2017)

21. Wang, Y.H., Xia, Y.H.: Strong convergence for asymptotically pseudocontractions with the demiclosedness principle in Banach spaces. Fixed Point Theory Appl. 1, 1-8 (2012)

22. Yao, Y.H., Liou, Y.C., Yao, J.C.: Split common fixed point problem for two quasi-pseudo-contractive operators and its algorithm construction. Fixed Point Theory Appl. 2015, 127, 19 pp. (2015)

23. Pan, C.J., Wang, Y.H.: Convergence theorems for modified inertial viscosity splitting methods in Banach spaces. Mathematics 7, 156 (2019)

\section{Submit your manuscript to a SpringerOpen ${ }^{\circ}$ journal and benefit from:}

- Convenient online submission

- Rigorous peer review

- Open access: articles freely available online

- High visibility within the field

- Retaining the copyright to your article

Submit your next manuscript at $\gg$ springeropen.com 